\title{
Investigations of Omega Precipitation in Titanium Molybdenum Alloys by Coupling 3D Atom Probe Tomography and High Resolution (S)TEM
}

\author{
A. Devaraj*, R. E. A. Williams**, S. Nag*, R. Srinivasan**, H.L. Fraser**, R. Banerjee* \\ * Center for Advanced Research and Technology and Department of Materials Science and \\ Engineering, The University of North Texas, UNT Research Park, Denton, Texas, 76207 \\ ** Center for the Accelerated Maturation of Materials and Department of Materials Science and \\ Engineering, The Ohio State University, 2041 College Road, Columbus, Ohio, 43210
}

Titanium-base alloys are used in a number of critical components in aerospace and defense, biomedical, automotive, and a range of other industries. These alloys typically exhibit complex multi-phase microstructures spanning across a range of length scales and also involving a large number of alloying additions. The $\omega$ phase is commonly observed in many commercial $\beta$ or near- $\beta$ titanium alloys on quenching from the solution treatment temperature in the single beta phase field [1]. These $\omega$ precipitates typically have an embrittling effect on the alloy and are therefore considered detrimental for its mechanical properties [2]. However, since $\omega$ precipitates are highly refined (nanometer scale) and homogeneously distributed, and due to the fact that they reject $\beta$-stabilizing elements, it is possible that they can act as heterogeneous nucleation sites for the precipitation of the equilibrium $\alpha$ phase. This leads to a homogeneous distribution of refined $\alpha$ precipitates that can substantially strengthen the alloy [1,3]. Therefore, the detailed investigation of $\omega$ precipitation in the beta matrix of titanium alloys is rather important.

This presentation investigates $\omega$ precipitation within the $\beta$ matrix of simple binary titanium molybdenum (Ti-Mo) alloys, by coupling 3D atom probe (3DAP) tomography and highresolution transmission electron microscopy (HRTEM) and HRSTEM. Quenching from $\beta$ solutionizing temperatures results in the formation of athermal $\omega$ precipitates that are typically considered to inherit the composition of the parent $\beta$ matrix [1]. On subsequent isothermal annealing, coarsening of the $\omega$ precipitates is accompanied by the diffusional rejection of Mo. While the as water-quenched Ti-9Mo (at\%) samples are nominally homogeneous in composition (shown by the atom map in Fig. 1(a)), a compositional profile across this reconstruction reveals modulations in the Mo content with a wavelength $\sim 3 \mathrm{~nm}$, indicating the early stages of phase separation (Fig. 1(b)). This is substantiated by the interconnected nature of the Ti=92at\% isoconcentration surface (isosurface) shown in Fig. 1(c). Electron diffraction patterns from the same sample exhibit reciprocal lattice streaking and intensity maxima at the $1 / 3$ and $2 / 3\{112\} \beta$ positions (inset in Fig. 1(d)), attributable to the early stages of $\omega$ precipitation in this alloy as clearly visible in the dark-field TEM (Fig. 1(d)). Isothermal annealing of this alloy, carried out for different periods of time at $475^{\circ} \mathrm{C}$, leads to growth and coarsening of the $\omega$ precipitates accompanied by the rejection of Mo from these precipitates. 3DAP results from a sample annealed for 0.5 hours is shown in Fig. 2. Fig. 2(a) shows a $\mathrm{Ti}=92$ at $\%$ isoconcentration surface (or isosurface), clearly delineating the $\omega$ precipitates, together with the Mo atoms in red. A darkfield TEM image from this sample (Fig. 2(b)), shows coarsened $\omega$ precipitates (diffraction pattern inset). A high-resolution TEM image of the $\beta / \omega$ interface, shown in Fig. 2(c), shows ledges present at this interface, suggesting a displacive component in the isothermal $\beta$ to $\omega$ transformation. 


\section{References}

[1] M. J. Blackburn and J. C. Williams, Trans. Met. Soc. AIME, 242, 2461 (1968).

[2] T.W. Duerig, and, J.C. Williams, Beta Titanium alloys in the 80's: Proceedings of the Symposium, Atlanta, GA, United States, 19-67 (1984).

[3] G. M. Pennock, H. M. Flower, and, D. R. F. West, Titanium '80: Science and Technology, 1343-1351 (1980).
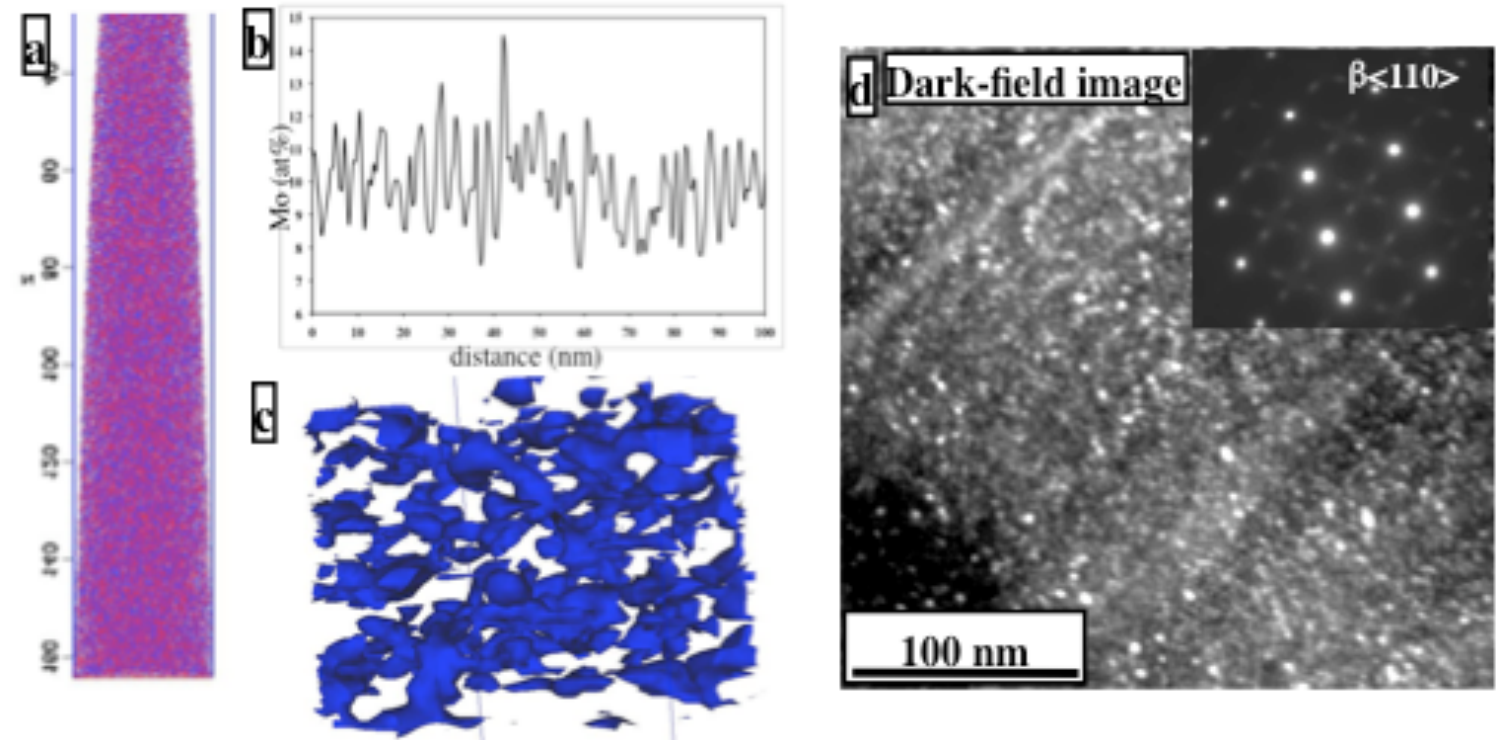

Fig. 1(a) 3DAP reconstruction of Ti(blue) and Mo(red) atoms in the Ti-9Mo alloy after beta solutionizing and quenching. (b) Mo compositional profile across the reconstruction shown in (a). (c) $\mathrm{Ti}=92 \mathrm{at} \%$ isosurface in blue. (d) Dark-field TEM image showing nanoscale omega precipitates in the same alloy together with diffraction pattern (inset).
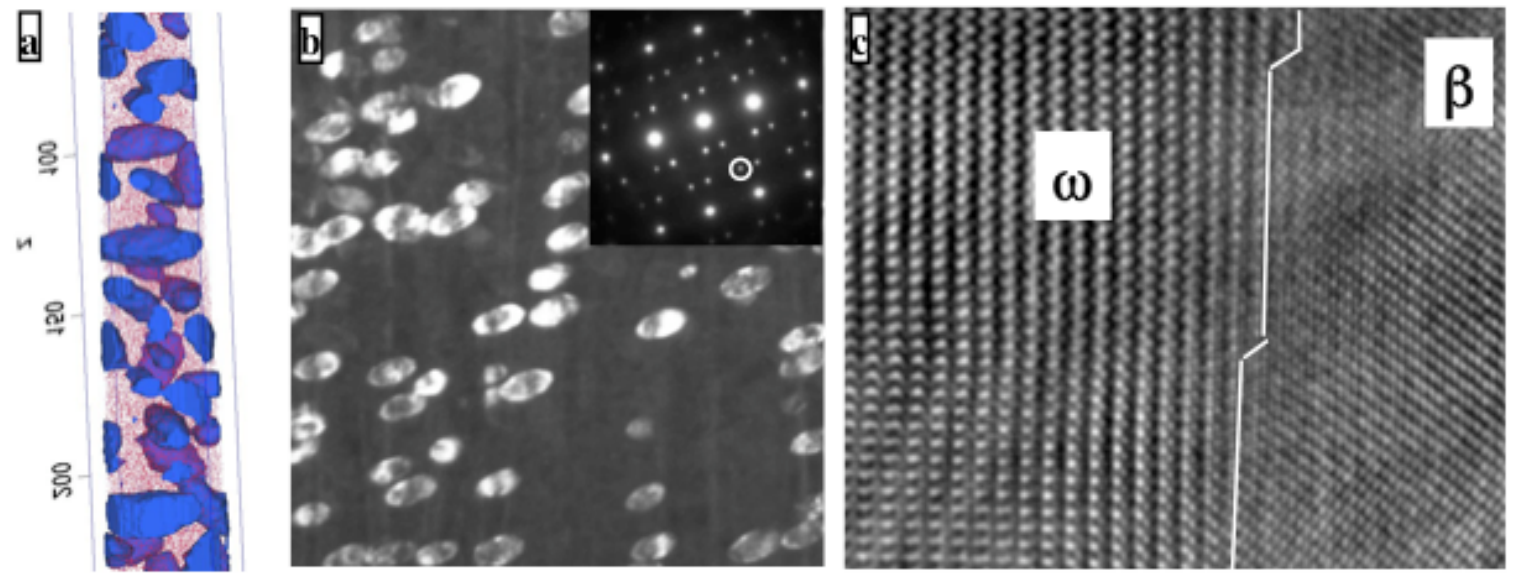

Fig. 2(a) 3DAP reconstruction of $\mathrm{Ti}=92 \mathrm{at} \%$ isosurface (blue) and $\mathrm{Mo}(\mathrm{red})$ atoms in the Ti-9Mo alloy after annealing at $475^{\circ} \mathrm{C}$ for $0.5 \mathrm{hrs}$. (b) Dark-field TEM image showing nanoscale omega precipitates in the same alloy together with diffraction pattern (inset). (c) HRTEM image of $\beta / \omega$ interface showing ledges. 